IOSR Journal of Pharmacy

e-ISSN: 2250-3013, p-ISSN: 2319-4219, www.iosrphr.org

Vol. 2, Issue 6, Nov-Dec. 2012, PP. 23-28

\title{
Dietary supplements and their role in obesity prevention
}

\author{
Vladimir Sadovoy ${ }^{1}$, Arcady Silantyev ${ }^{2}$, Olga Bunina $^{3}$, Tatiana Shchedrina ${ }^{1}$, \\ Anna Aralina ${ }^{3}$ \\ ${ }^{l}$ North-Caucasian Federal University, Pyatigorsk, Russia, \\ ${ }^{2}$ Povolzhskiy State University of Telecommunications and Informatics, Stavropol, Russia, \\ ${ }^{3}$ Stavropol State Agrarian University, Stavropol, Russia,
}

\begin{abstract}
In this study, the molecular properties of L-carnitine and lecithin were investigated by applying computational chemistry methods. Molecular docking method provided rationale for using these agents as metabolic and lipolytic activators affecting intestinal lipid digestion and blocking fat formation. Prophylactic properties of lecithin and L-carnitine were confirmed by testing the effects of prototype lipid formulations consisting of homogenized water-in-oil mixture on experimental animals.
\end{abstract}

Keywords: lecithin, L-carnitine, lipase, molecular properties, molecular docking, complex molecular compounds.

\section{INTRODUCTION}

The World Health Organization (WHO) estimates that more than a billion people in the world are suffering from excess weight. In the developed countries, the body mass of approximately $30 \%$ of the population exceeds the norm by $20 \%$ or more. Obesity is a risk factor for atherosclerosis, hypertensive disease, diabetes and other illnesses. It has been established that an excess body weight of $10 \%$ increases mortality by the average of $30 \%$. In Russia, $25 \%$ of the working-age population are suffering from obesity, and $30 \%$ are overweight [1]. These data show that obesity should be considered as a salient social issue. Prevention may help avoid the spread of obesity.

The first step in excessive body weight prevention should be switching to a more balanced diet, making it more beneficial for one's health. Using dietary supplements and functional foods is seen among the more effective weight-reducing strategies. Dietary supplements are natural or synthesized substances (or their concentrates) that do not contain medicinal ingredients and designed to be taken with meals or integrated into foods [2].

One of the focus areas in creating new functional foods for the prevention of obesity is developing processing technologies for food products enriched with dietary supplements. In this regard, the component composition of the raw materials used is of crucial importance, as it enables the regulation of the finished products' nutritional value and their functional and technological properties, as well as the prognosis of the expected effect.

In clinical practice, the following types of dietary supplements are used for prevention and treatment of obesity: metabolic and lipolytic activators, fat formulation inhibitors, agents that reduce intestinal fat digestion and have other therapeutic and prophylactic properties.

In obesity prevention, studying the metabolism and lipolysis activation mechanism and the effect of dietary supplements on intestinal fat digestion is of special interest. For these purposes, the most commonly used supplements are L-carnitine and lecithin. These supplements are also known to have multifaceted therapeutic and prophylactic properties and be beneficial for human health.

Knowledge of the spatial structure of the complexes of lipotropic fatty acids compounds and enzyme systems interactions with ligands is an important step towards understanding the mechanisms of their functioning. Rational design of new food products for people suffering from excessive body weight requires structural information about the interactions between lipolysis (fats breakdown under the influence of lipase) activators and fat formulation and digestion inhibitors.

\section{EXAMINATION OF MOLECULAR PROPERTIES OF DIETARY SUPPLEMENTS}

At the initial stage, the charge distribution density and molecular properties of L-carnitine, fatty acid (Fig. 1, 2) and lecithin were examined to assess the capability for the formation of complex compounds. As fatty acids are monobasic aliphatic compounds containing a linear chain and differing only in the number of carbon atoms, the modeling of molecular properties was conducted using oleic acid (molecular weight $282.47 \mathrm{amu}$ ). 
Dietary supplements and their role in obesity prevention

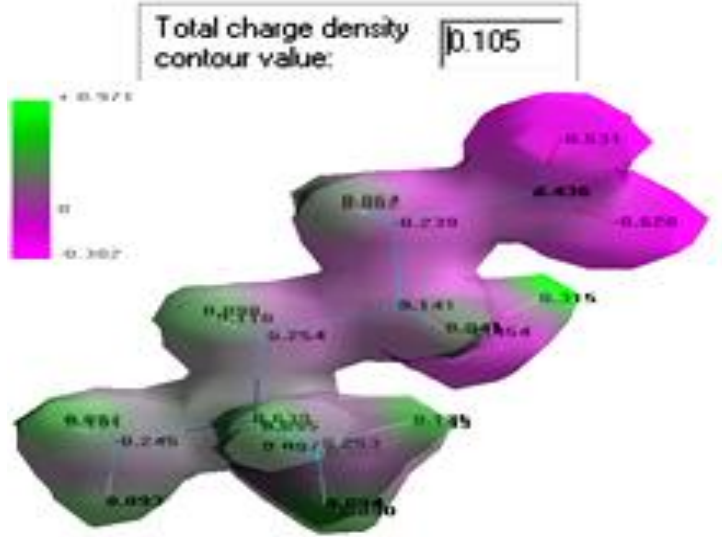

a)

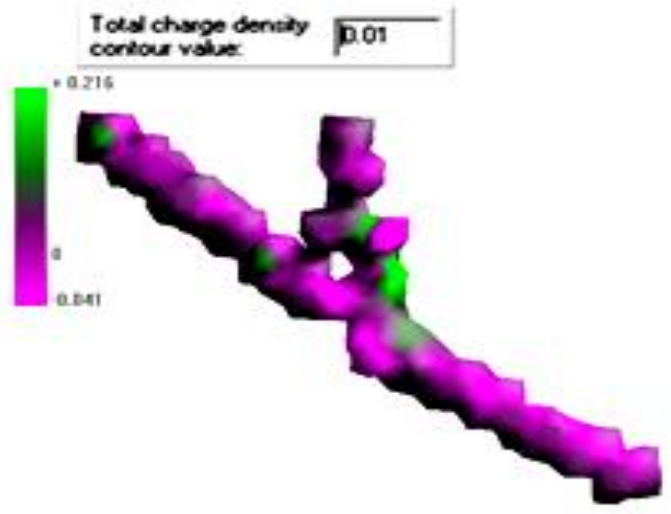

c)

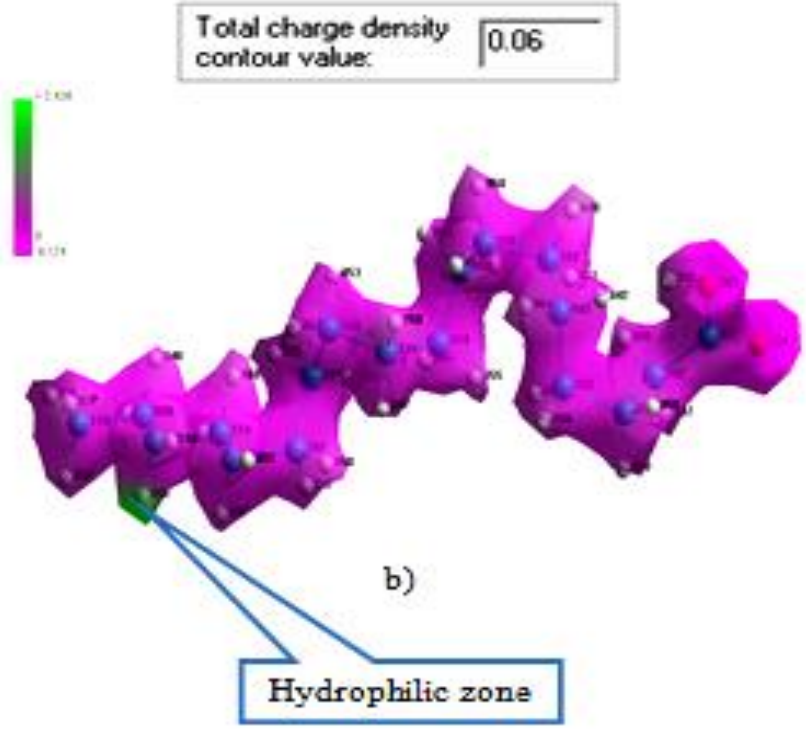

Figure 1 - Charge distribution density in the molecules of

a) L-carnitine,

c) lecithin

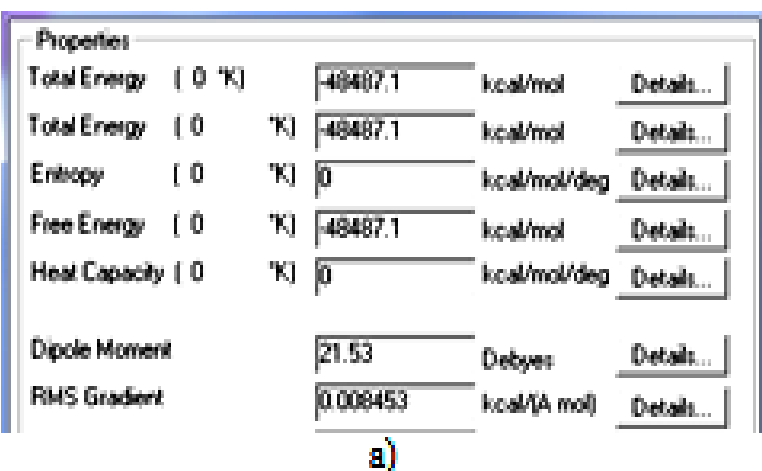

\begin{tabular}{|c|c|c|c|c|}
\hline \multicolumn{3}{|l|}{ Pisperisi - } & \multirow{3}{*}{$\begin{array}{l}\text { kcal/mol } \\
\text { keal/mol }\end{array}$} & \multirow{3}{*}{$\begin{array}{l}\text { Detoll... } \\
\text { Dotolit... }\end{array}$} \\
\hline Toud Ereigy $\quad\left(\begin{array}{lll}0 & 0 & \mathrm{x}\end{array}\right)$ & & 76.951 & & \\
\hline Total Enesgy 10 & $\mathrm{x}_{\mathrm{j}}$ & 75851 & & \\
\hline Enthopy & K] & 0 & healmoldog & Detall.. \\
\hline Fiee Energy 10 & ${ }^{6} \mathbf{j}$ & $\sqrt{3651}$ & $\mathrm{kcal} / \mathrm{mol}$ & Deladt... \\
\hline Hew Caposity I 0 & 'K] & 0 & $k \cos / \mathrm{mol} / \mathrm{deg}$ & Detall... \\
\hline Decole Momers & & 0 & Debyes & Detall... \\
\hline AMS Gradeenk & & 02843 & kealat mol] & Dotalt... \\
\hline
\end{tabular}

c)

\begin{tabular}{|c|c|c|c|c|}
\hline \multicolumn{3}{|l|}{ Propention- } & \multirow{3}{*}{ kcal/mol } & \multirow{3}{*}{ Detalk... } \\
\hline Tasl Enowgy $\left[\begin{array}{ll}0 & \mathrm{~K}\end{array}\right]$ & & 225121 & & \\
\hline Tas Enogy 10 & $\left.{ }^{2} \mathrm{k}\right)$ & 22512 & & \\
\hline Entopy & $\mathrm{K}]$ & 0 & $\mathrm{kes} / \mathrm{mol} / \mathrm{deg}$ & Detalna.. \\
\hline Fiee Eneigy 10 & $\mathrm{k}$ & 225121 & $\mathrm{kes} / \mathrm{mol}$ & Detalna.. \\
\hline Hex Cosesty 10 & $\left.{ }^{2} \mathrm{~K}\right]$ & 0 & hcal/mol/deg & Detolli... \\
\hline Depole Noments & & 3574 & Debyes & Detosid... \\
\hline AMS Gradent & & 00173 & kealla moll & Oetalin... \\
\hline
\end{tabular}

b)
Figure 2 - Molecular properties of L-carnitine (a), oleic acid (b) and lecithin (c) 
The results indicated that the molecule of L-carnitine (Fig. 1 a) has pronounced hydrophilic properties, as evidenced by the color pattern of the illustration, the total charge density $(0.105 \mathrm{eV})$, and the dipole moment $(21.53 \mathrm{D})$. The molecular surface of oleic acid (Fig. 1b) is hydrophobic (total charge density equals $0.06 \mathrm{eV}$, the dipole moment - 3.574 D), with is only one small segment having the hydrophilic properties. Despite its low total charge density $(0.01 \mathrm{eV})$, the lecithin molecule has hydrophilic properties and has significant hydrophobic segments. The dipole moment which characterizes the properties of the dipole (according to the charge distribution on the van der Waals radius) has a sufficiently low value ( 0 - for lecithin molecules.) These findings indicate that lecithin: is a surface-active agent with high hydrophobic and hydrophilic activity levels, produces stable emulsions, and is effective in phase interface of various substances.

The potential energy of the molecules confirms that oleic acid is an unstable compound (Total Energy equals $22.5121 \mathrm{kcal} / \mathrm{mol}$ ) involved in the energy process which leads to ATP synthesis (Fig. 2b). L-carnitine molecule (Fig. 2a) is a stable compound (Total Energy - $48487.1 \mathrm{kcal} / \mathrm{mol}$ ) and can participate in the subsequent fatty acids transformation cycles. The quadratic gradients of the molecules indicate that the process of geometric optimization of the molecular structures was carried out properly.

Thus, the quantum chemical molecular analysis has shown that L-carnitine and lecithin have strong hydrophilic properties, and the oleic acid molecule is hydrophobic (with one small segment which has hydrophilic properties). The performed geometric optimization of the molecular structure and molecular properties analysis are necessary for applying the molecular docking method to the study of the complex compound formation process.

\section{COMPLEX COMPOUND ANALYSIS}

The lipotropic effects of dietary supplements and the influence they have on intestinal fat digestion can be assessed by examining the potential for the complex compound formation.

For the purposes of lipotropic effect assessment (digestion and transport of fats) utilizing the computational chemistry methods, molecular docking of L-carnitine and oleic acid was performed. Molecular docking is used to predict such position and orientation of molecules that would be the most beneficial in formatting a stable compound. Docking was performed using the simulation method, where the receptor and the ligand are separated by a certain physical distance, and the ligand finds its position into the protein's active site after a certain number of "steps". These steps include the transformations undergone by the studied molecules, their shifting and rotation, as well as the internal structural changes of the ligand, including its angular rotations. Each of these spatial steps changes the system's total energy assessment, which is calculated after each step [3].

The results of molecular docking of oleic acid to L-carnitine confirm the lipotropic effect of the complex compound formation. The intermolecular binding energy was established at $-89.08 \mathrm{kkal}$, which indicates the sufficiently high molecular compatibility. It was also found that L-carnitine and human pancreatic lipase (information resources of the Protein Data bank *.pdb) form a stable ligand-receptor compound with low intermolecular binding energy $(-117.91 \mathrm{kkal})$; however, the active lipase center is not blocked in created compound, which means that the enzyme will continue functioning as the lipid hydrolysis catalyst (fig.3).

The molecular interaction between lecithin and human pancreatic lipase was analyzed using the AutoDock application. Figure 4 shows pancreatic lipase and lecithin prior to molecular docking. The lipase molecule clearly exhibits the active center.

The results of the molecular docking (fig.5) indicated that a single lecithin molecule is insufficient to effectively block the energetic (active center) zone of the pancreatic lipase, despite forming a stable complex compound. The enzyme's active remains functional.

Two lecithin molecules, on the other hand, create the full blockage of the active center of pancreatic lipase, forming a highly stable compound with intermolecular binding energy of $-412.36 \mathrm{kkal}$. As shown in Figure 5., the active center ceases to exist after the docking.

The molecular-dynamic calculations performed in this study show that lecithin blocks the active center of pancreatic lipase, thus preventing the enzymatic hydrolytic breakdown of lipids and their digestion by human organism.

The degree of lipid hydrolysis can be characterized by the acid value, which is used to measure the quantitative content of free fatty acids. To assess the effectiveness of dietary supplements, experimental lipid compositions were formulated by adding lecithin-containing agents to water-in-oil homogenized emulsion. The dietary supplements' ratio in the compositions was determined according to their quantitative proportion in the physiological reference nutrient intakes.

During the pilot studies, pharmaceutical pancreatin was used as the enzymatic agent. With the active acidity of the lipase function found to lie within the optimum range $8-9$, the $\mathrm{pH}$ balance of the water-in oil mixture was regulated with the $0.1 \mathrm{~N}$ sodium hydroxide solution. The resulting mixture was thermostated at $37^{\circ} \mathrm{C}$; at specified intervals (30 min.) the mixture was sampled for determining acid value.

After two hours of enzymatic treatment, the water-in-oil homogenized mixture with added lecithin agent was found to have a lower acid value (11.91) compared to that of the proof sample formulated without 
Dietary supplements and their role in obesity prevention

adding diet supplements (77.87 $\mathrm{mg} \mathrm{KOH} \mathrm{/} \mathrm{g} \mathrm{oil).} \mathrm{Lecithin} \mathrm{and} \mathrm{allicin} \mathrm{were} \mathrm{determined} \mathrm{to} \mathrm{lower} \mathrm{the} \mathrm{efficiency} \mathrm{of}$ pancreatic lipase. In addition, the lipase was found to exhibit the highest activity during the first 30 minutes of enzymatic hydrolysis.
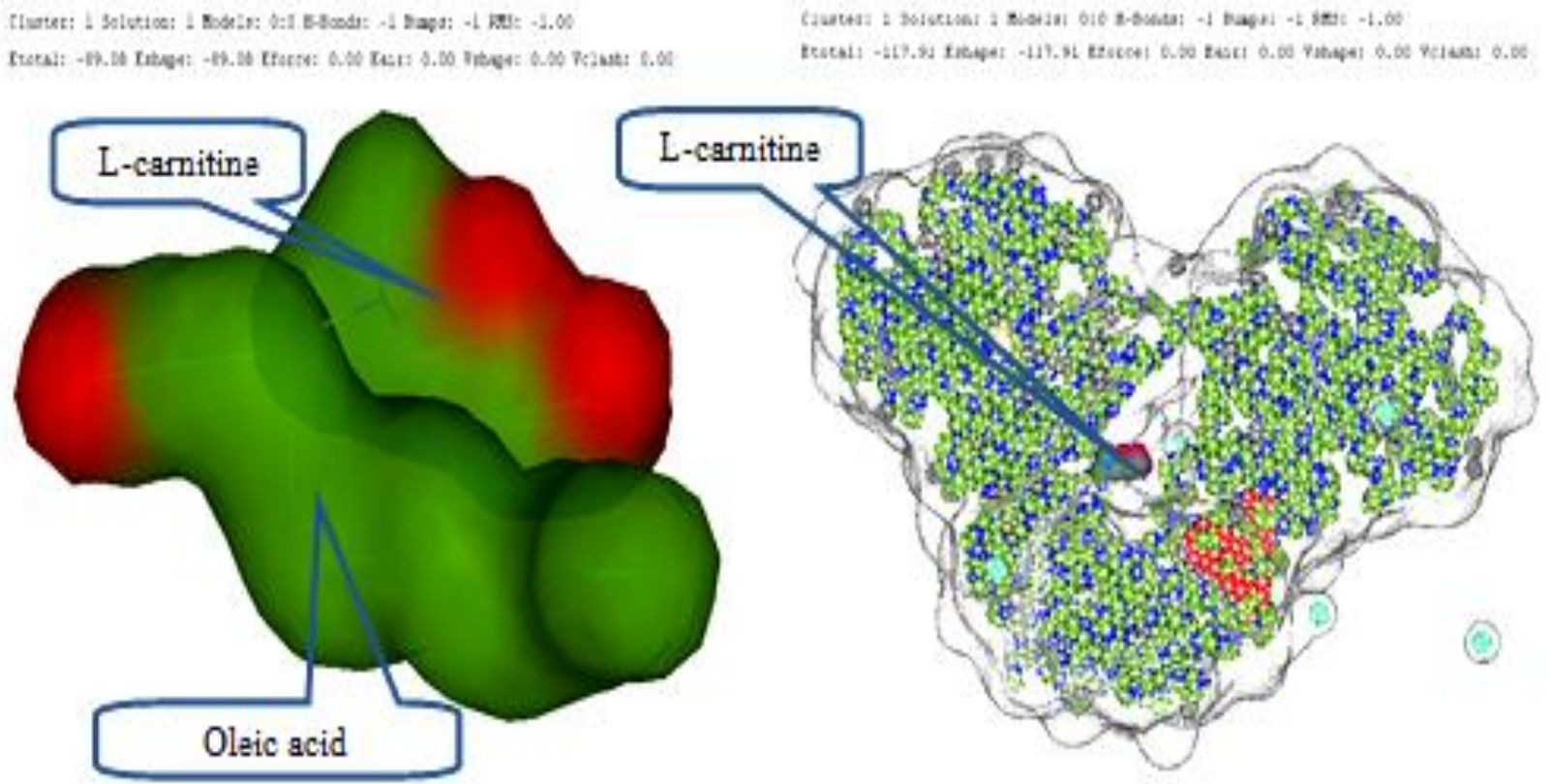

a)

b)

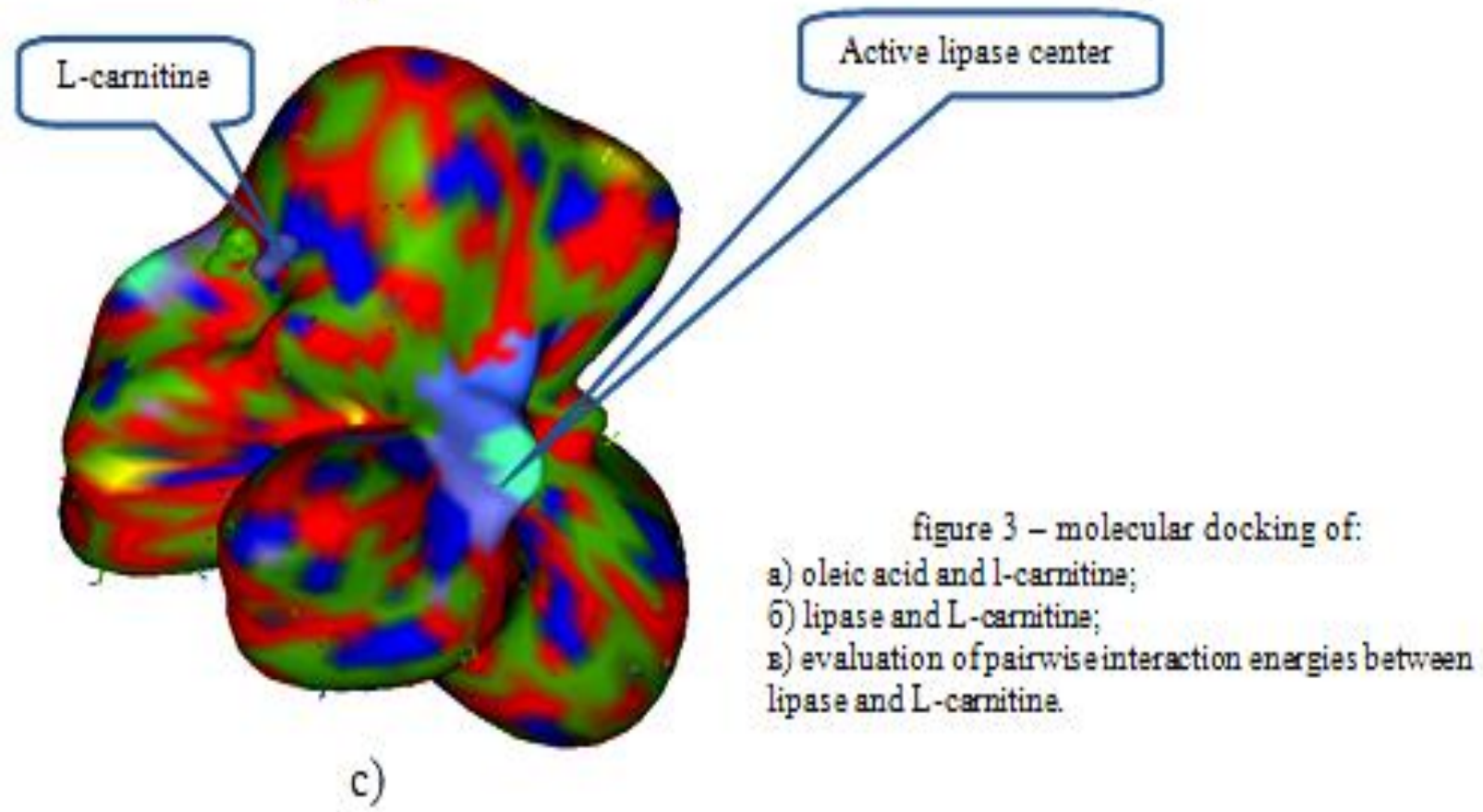

The formulation and technology for production of a lecithin-containing dietary supplement was created, based on the study materials for the enzymatic hydrolysis of a lipid fraction with lipase inhibitors, and in compliance with the reference nutrient intake of the components used. The supplement's oxidative and microbial spoilage parameters were within the requirements of sanitary regulations and norms № 2.3.2.1078 01 .

To assess the prophylactic and dietetic properties of the dietary supplement test sample, obesity was simulated in experimental animals by increasing their caloric intake through introducing excessive amounts of lipids into their diet. The experiments were performed on white mice weighing $20-30 \mathrm{~g}$, with individual weight in the groups varying from $10 \%$ to $15 \%$. The research has shown that a diet that includes a supplement with selective active ingredients can be used not only preventatively, but also for treating various illnesses. The 
experimental supplement's two active ingredients, essential L-carnitine and lecithin, reduce obesity and normalize lipid exchange and carbohydrate, protein and mineral metabolism, even for the organisms with excess body

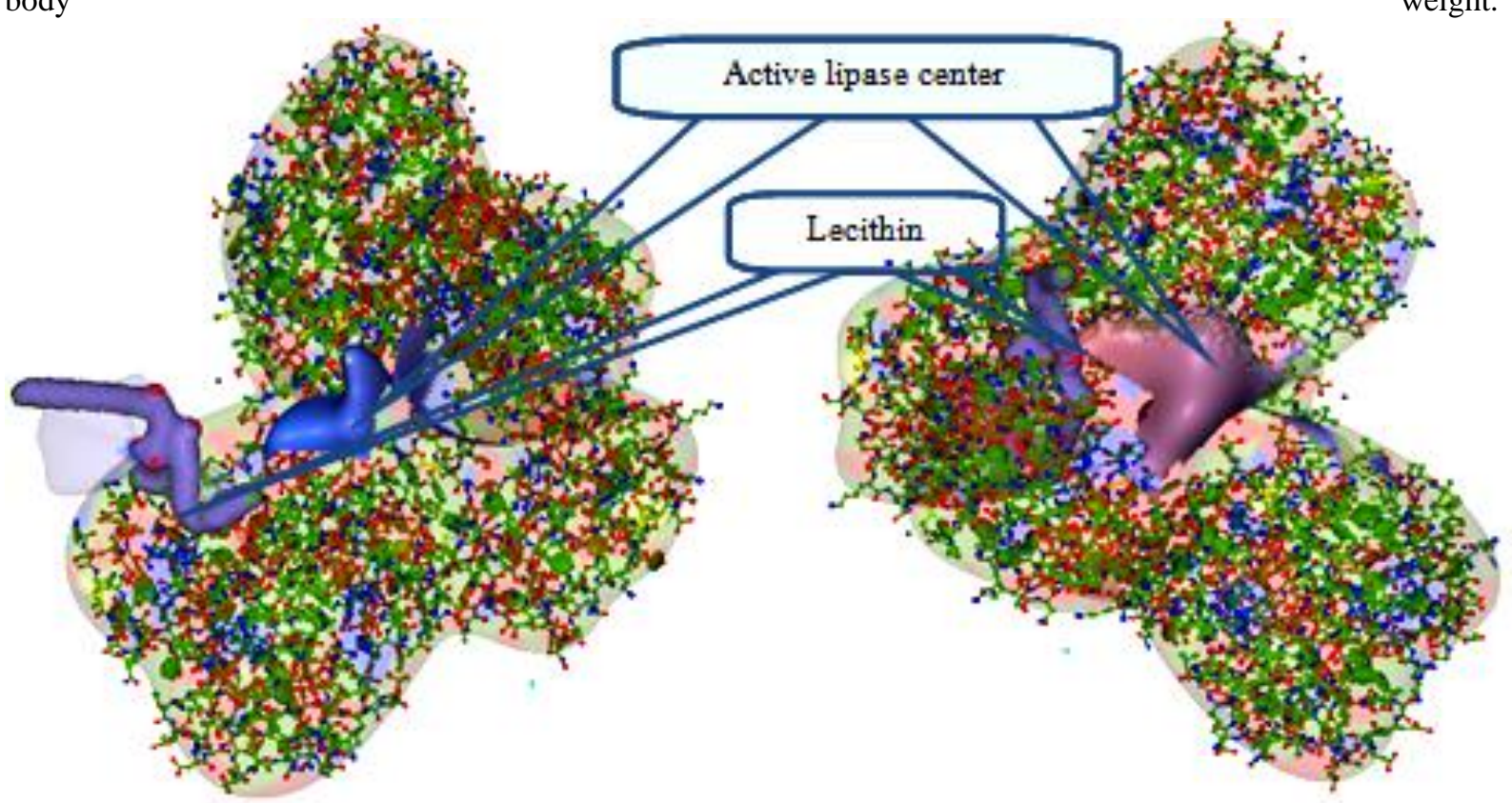

a)

b)

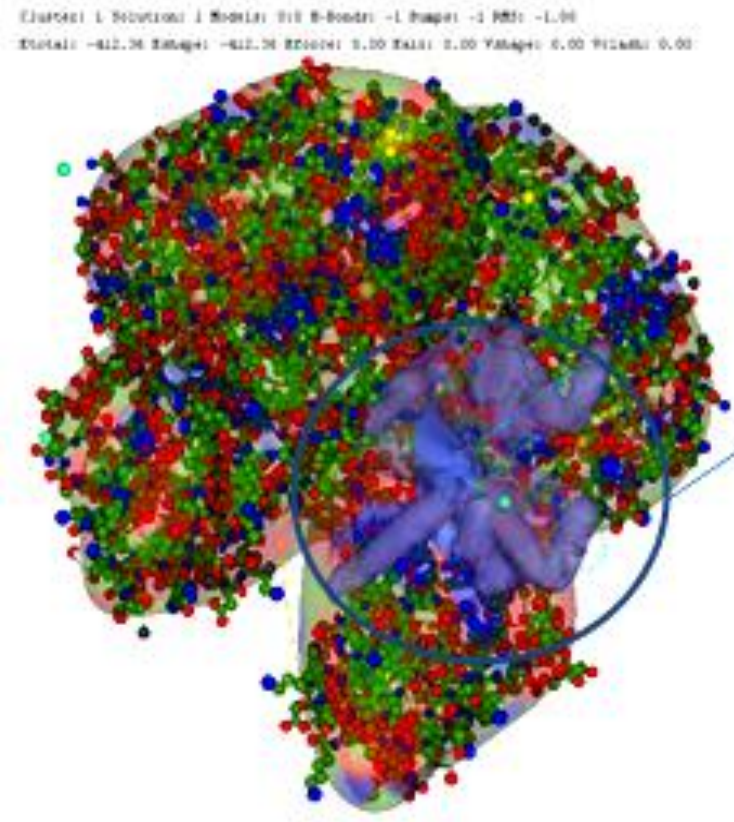

c)

\section{Two lecithin molecules}

Figure 3.6 - A study of pancreatic lipase exposed to lecithin and allicin:

a) pancreatic lipase prior to molecular docking:

b) pancreatic lipase after docking with 1 lecithin molecule;

c) pancreatic lipase after docking with 2 lecithin molecules;

\section{CONCLUSION}

The work performed has shown that L-carnitine and lecithin have strong hydrophilic properties. So two lecithin molecules create the full blockage of the active center of pancreatic lipase, forming a highly stable compound with intermolecular binding energy of $-412.36 \mathrm{kkal}$. Hypothesis that a diet including a supplement with selective active ingredients can be used preventatively was verified in experiment on animals. We suppose as a perspective of this research program also treating various illnesses. 


\section{SOURCES}

1) Ustinova A. V., Byelyakina N. E., Morozkina I. K. et.al. Lower Calorie Semi-Processed Foods for Prophylactic Diet Of Adults and Children // Moscow: Meat industry. - 2004. -- № 3. - pp. 22-25.

2) Tutyelyan V. A., Spiritchev V. B., Sukchanov B. P., Kudasheva V. A. Micronutrients in the Diet of A Healthy and a Disease-Affected Person: a guide to vitamins and minerals. - Moscow: Kolos, 2002. $424 p$.

3) Kosinsky J. A., Pyrkov T. V., Lutcenko S. V., YefremovR. G. Structural Prognosis Of Ligand-Protein Compunds: From a Computer Model to Biological Function. // Moscow: Russian Chemistry Journal. 2006. -№ 2. - pp. 36-44. 